\title{
Mapping the spatial proteome of metastatic cells in colorectal cancer
}

Marta Mendes ${ }^{\ddagger}$, Alberto Peláez-García ${ }^{\ddagger}$, María López-Lucendo ${ }^{\ddagger}$, Ruben A. Bartolomé $^{\ddagger}$ Eva Calviño ${ }^{\ddagger}$, Rodrigo Barderas ${ }^{\ddagger * *}$, J. Ignacio Casal ${ }^{* * *}$

${ }^{\ddagger}$ Department of Cellular and Molecular Medicine, Centro de Investigaciones Biológicas (CIB-CSIC), Madrid, Spain

${ }^{\S}$ Instituto de Salud Carlos III. Majadahonda. Spain

IIPresent address: Technische Universität Berlin, Germany

${ }^{¥}$ Present address: Department of Pathology, Hospital Universitario La Paz, IdiPAZ, Madrid, Spain

Running title: Mapping the metastatic proteome

Keywords: Spatial proteome, cell fractionation, SILAC, metastasis, colorectal cancer

** To whom correspondence should be addressed:

Department of Cellular and Molecular Medicine, Centro de Investigaciones Biológicas (CIB-CSIC), Ramiro de Maeztu, 9, 28040 Madrid, Spain. Tel.: +34 918373112; E-mail: icasal@cib.csic.es.

Instituto de Salud Carlos III, Majadahonda, Spain Carretera de Majadahonda - Pozuelo, Km. 2.200, 28220 Majadahonda, Madrid, Spain. Tel.: +34 913944155; E-mail: r.barderasm@isciii.es 


\section{ABSTRACT}

Colorectal cancer (CRC) is the second deadliest cancer worldwide. Here, we aimed to study metastasis mechanisms using spatial proteomics in the KM12 cell model. Cells were SILAC-labeled and fractionated into five subcellular fractions corresponding to: cytoplasm, plasma, mitochondria and ER/golgi membranes, nuclear, chromatin-bound and cytoskeletal proteins and analyzed with high resolution mass spectrometry. We provide localization data of 4863 quantified proteins in the different subcellular fractions. A total of 1318 proteins with at least 1.5 -fold change were deregulated in highly metastatic KM12SM cells respect to KM12C cells. The protein network organization, protein complexes and functional pathways associated to CRC metastasis was revealed with spatial resolution. Although $92 \%$ of the differentially expressed proteins showed the same deregulation in all subcellular compartments, a subset of 117 proteins $(8 \%)$ showed opposite changes in different subcellular localizations. The chaperonin CCT, the Eif2 and Eif3 initiation of translation and the oxidative phosphorylation complexes together with an important number of guanine nucleotidebinding proteins, were deregulated in abundance and localization within the metastatic cells. Particularly relevant was the relationship of deregulated protein complexes with exosome secretion. The knowledge of the spatial proteome alterations at subcellular level contributes to clarify the molecular mechanisms underlying colorectal cancer metastasis and to identify potential targets of therapeutic intervention. 


\section{Significance of the study}

Metastasis is responsible for $90 \%$ of the cancer-associated deaths. To understand underlying cellular mechanisms that favor metastasis is essential to discover new biomarkers and therapeutic strategies. Here, we have performed a multidimensional quantitative proteomic analysis to identify not only those differences in protein abundance between metastatic and non-metastatic cells, but also differences in subcellular location. An exhaustive analysis of the quantified proteome with different bioinformatics tools enabled the characterization of large macromolecular complexes and pathways alterations in metastatic cells. In addition, we have identified a number of protein complexes that showed disparities in the expression levels between different locations in metastatic versus non metastatic cells. These alterations play a significant role in cellular processes such as adhesion, migration and vesicle trafficking. We hypothesized that vesicle trafficking alterations must be related with the increase in exosome secretion characteristic of the metastatic cells. Finally, some potential targets of therapeutic intervention have been identified. 


\section{INTRODUCTION}

Current proteomic strategies use a monodimensional bottom-up workflow where quantitative data are averaged between different cellular pools. Despite its relevance, the single dimension analysis of the proteome misses very relevant functional information. Protein properties, including abundance levels, protein-protein interactions, post-translational modifications (PTMs), subcellular localization patterns and protein synthesis and degradation rates, are all highly dynamic and can change rapidly between different compartments during the course of different biological processes, such as cell proliferation, cell migration, differentiation and metastasis [1]. Spatial proteomics, which studies organelle proteomics and the dynamics of subcellular distribution of proteins in response to a variety of factors, is a rapidly growing field [2-5]. Changes in the spatiotemporal subcellular distribution of proteins have been detected in many cell types in response to environmental stress, cell-cycle signals, growth factors, or hormone exposure [1, 6-9]. Indeed, changes in subcellular localization may affect their cellular functions of proteins and their capacity to interact with other proteins. Protein alterations in localization are critical in disease [10], as proteins at different subcellular localizations possess different functional roles $[2,11,12]$. It is well established that loss- or gain-of-function of proteins in many diseases could be attributed to unexpected protein subcellular localizations $[13,14]$.

Despite recent advances in diagnosis and therapy, colorectal cancer (CRC) remains as the second deadliest cancer in developed countries [15]. The identification of metastasis-associated proteins and altered pathways underlying CRC metastasis would contribute to discover new targets and to improve patient survival. Metastasis is a multistage process that implies adaptation of cells to distant microenvironments beyond the primary tumor $[16,17]$. For this study, we used the isogenic KM12 cell model 
consisting of KM12SM and KM12C cells [18]. KM12SM CRC cell line with high metastatic capacity was isolated from liver metastases in nude mice after five cycles of intrasplenic injection of the poorly metastatic KM12C cell line. In previous reports, we analyzed alterations in protein expression in the secretome and the plasma membrane of this cell system by antibody microarrays and quantitative proteomics, respectively [1921].

Here, KM12 cells were metabolically labelled using SILAC. SILAC has been successfully used in spatial proteomics for the analysis of proteome turnover and for the identification of changes in proteome localization as a consequence of DNA damage [9, 22, 23]. Subcellular fractionation of the KM12 contributed to clarify the molecular mechanisms underlying CRC metastasis. Protein abundance and localization were measured in parallel for the five separate subcellular fractions, providing a draft map of the spatially deregulated protein complexes and networks in CRC metastasis. 


\section{Materials and Methods}

\subsection{Cell culture, labeling and subcellular fractionation}

Human colon cancer cells KM12C and KM12SM were provided by Dr Fidler's laboratory (MD Anderson Cancer Center, TX, USA) [18, 24]. DMEM (Dulbecco's modified Eagle's medium) supplemented with L-lysine and L-arginine (light medium) or $\left[{ }^{13} \mathrm{C}_{6}\right]$-L-lysine and $\left[{ }^{13} \mathrm{C}_{6}\right]$-L-arginine (heavy medium) was purchased from Dundee Cell Products. Both cell lines were labeled with heavy and light medium to perform label swapping SILAC experiments as biological and technical replicates, as previously described [20, 25]. After 8 cell doublings to ensure $>98 \%$ incorporation of labeled amino acids, cells were resuspended with $4 \mathrm{mM}$ EDTA PBS and washed with ice-cold PBS prior to fractionation with the Subcellular Protein Fractionation Kit for Cultured Cells (Pierce). Cells were divided into five subcellular fractions corresponding to cytoplasm (CEB), plasma, mitochondria and ER/golgi membranes (MEB), nuclear soluble (NEB), nuclear chromatin-bound (NEB-CBP) and cytoskeletal proteins (PEB).

\subsection{SDS-PAGE and protein digestion}

Protein concentration after subcellular fractionation was quantified using the 2D Quant Kit (GE Healthcare). Then, $25 \mu \mathrm{g}$ of each subcellular fraction was separated by SDS PAGE. Gels were stained with Colloidal Blue Staining Kit (Invitrogen). Lanes containing forward and reverse SILAC experiments for each fraction were cut into 20 slices that were further cut in small pieces prior to in-gel digestion with trypsin. Peptides were extracted using 100\% ACN and 0.5\% TFA. Extracted peptides were dried and purified using a C18 resin Zip Tip (Millipore). Finally, samples were reconstituted in $5 \mu 10.1 \%$ formic acid/2\% ACN before LC-MS/MS. 


\subsection{LC-MS/MS and data analyses}

Peptides were trapped in a C18-A1 ASY-Column 2-cm precolumn (Thermo Scientific) and eluted to a $\mathrm{C} 18$ reverse phase column $(15 \mathrm{~cm}$ x $75 \mu \mathrm{m}, 3 \mu \mathrm{m}$ particle Nanoseparations), where they were separated using a 150 min gradient from 0 to $35 \%$ buffer B (Buffer A: 0.1\% formic acid/2\% ACN; Buffer B: 0.1\% formic acid in ACN) at a flow rate of $250 \mathrm{nl} / \mathrm{min}$. A NanoEasy HPLC (Proxeon) was coupled to an LTQ Orbitrap Velos (Thermo Scientific) equipped with a nano-electrospray ion source (Proxeon). The LTQ Orbitrap Velos was operated in positive mode and data-dependent manner. Full scan MS spectra (m/z 400-1200) were acquired using a target value of $10^{6}$ and a resolution of 60000 at $\mathrm{m} / \mathrm{z}$ 400. The top 15 ions were selected for fragmentation in the linear ion trap using a target value of $10^{4}$ and a normalized collision energy of $35 \%$. Precursor ion charge state screening and monoisotopic precursor selection were enabled and singly charged ions and unassigned charge states were rejected. Dynamic exclusion was enabled and set up to 30 s and one repeat count.

MS data were analyzed and quantified using Proteome Discoverer (PD, version 1.3.0.339, Thermo Scientific). Proteins were identified by interrogating the human SwissProt Database (SwissProt_57.15.fasta) with Mascot (version 2.3, MatrixScience). Carbamidomethylation of cysteines was set as a fixed modification, while oxidation of methionine, N-terminal acetylation and $\left[{ }^{13} \mathrm{C}_{6}\right]-\operatorname{Arg},\left[{ }^{13} \mathrm{C}_{6}\right]$-Lys were set as variable modifications. Precursor and fragment mass tolerance were set to $10 \mathrm{ppm}$ and $0.8 \mathrm{Da}$, respectively, and a maximum of two missed cleavages were allowed. Identified peptides were filtered using Percolator algorithm with a q-value threshold of 0.01 and a Mascot significant threshold of 0.05 . The mass spectrometry data have been deposited in the ProteomeXchange Consortium via the PRIDE partner repository with the dataset identifier PXD006656. 
Only those proteins quantified with at least 2 unique peptides in both, forward and reverse, experiments were considered in the analysis. Protein ratios with variability greater than $20 \%$ were discarded from the analysis. All experiments were corrected by normalizing data against the $5 \%$ trimmed mean of the data set to center the $\log _{2}$ ratio distribution to zero [20]. As we previously described for KM12 cells, arginine to proline conversion did not affect quantification accuracy [20]. A 1.5-fold change cut-off for deregulated proteins was determined to be significant using a permutation-based test $[25,26]$.

\subsection{Western blot analysis}

Ten $\mu \mathrm{g}$ of each fraction were separated by $10 \%$ SDS-PAGE and directly transferred to Hybond-C nitrocellulose membranes (GE Healthcare) using mini trans-blot equipment (Bio-Rad). Membranes were blocked and incubated with optimized dilutions of monoclonal and polyclonal primary antibodies (Table S1) followed by either HRP antimouse IgG (Pierce) or HRP anti-rabbit IgG (Sigma-Aldrich) at a 1:5000 dilution. Proteins were visualized with ECL SuperSignal West Pico (Pierce). The abundance of the proteins in western blot assays was determined by densitometry using Quantity One 1D Analysis Software v4.6 (Bio-Rad Laboratories).

\subsection{Confocal microscopy}

KM12SM cells were cultured on glass coverslips pre-treated with Matrigel for $24 \mathrm{~h}$. They were fixed in a fresh solution of $4 \%$ paraformaldehyde in PBS for 15 min and then permeabilized with Triton X-100 1\% in PBS for 10 min. After two washes with PBS, cells were incubated with the indicated primary antibodies for $1 \mathrm{~h}$ at 1:50/1:100 dilutions (Table S1), followed by incubation with Alexa-488 labelled secondary 
antibodies for $35 \mathrm{~min}$, phalloidin coupled to Alexa 568 and 4,6-diamidino-2phenylindole (DAPI). Samples were mounted with Mounting Fluorescence Medium (Dako, Copenhagen, Denmark). Images used for protein localization were acquired at room temperature using a Leica TCS-SP5-AOBS microscope equipped with a $63 \times$ oilimmersion objective.

\subsection{Bioinformatics and statistical analysis}

Subcellular classification and significant GO terms were annotated by PANTHER, as determined by Fisher's exact test $(p$ value $\leq 0.05)$ [27]. To identify CRC metastasis specific alterations, bioinformatic analysis was performed using DAVID, KEGG, STRING and IPA (Qiagen Bioinformatics) to identify altered protein networks and pathways [28-32]. For protein-protein interaction analysis we used STRING. A circular layout algorithm was used to minimize overlapping edges and facilitate the visualization of the interaction between the nodes [33]. Significant subnetworks were shown as inner circles within the global networks. Cytoscape was used for network representation and visualization [34]. Unsupervised cluster analysis was performed with MeV [35]. 


\section{RESULTS}

\subsection{Subcellular fractionation and differential protein expression in metastatic}

KM12 cells

Forward and reverse SILAC-labeled KM12 cells were biochemically fractionated into five subcellular fractions - CEB, MEB, NEB, NEB-CBP and PEB- using a commercial kit based on the use of successive detergents and differential centrifugation (Fig. 1A). To confirm a correct fractionation, we investigated the quality of the different subcellular fractions using proteins of known subcellular localization (Rho-GDI, EGFR, SP1, Histone 3 and VIM) by western blot (Fig. 1B). After MS analysis, these control proteins showed the highest intensity at the same subcellular compartment than western blot. However, the detection of control proteins at low intensity in adjacent subcellular fractions confirmed some minor cross-contamination during cell partitioning (Fig. 1C).

We identified a total of 6420 proteins in the five subcellular fractions, corresponding to 4863 unique quantified proteins. A detailed description of identified and quantified peptides and proteins in all the subcellular fractions is shown in Fig. 2A (Tables S2, S3). From the total of 4863 proteins quantified, we observed the presence of 1453 proteins in only one compartment, 1590 proteins in two compartments, 1146 in three fractions, 559 in four, and 115 proteins ubiquitously distributed in the five compartments (Fig. 2B). As another test to confirm a correct cellular fractionation, we carried out a GO classification of the identified proteins in each of the 5 subcellular fractions (Fig. 2C). In all fractions, the quantified proteins were correctly classified within the three first GO classification hits of each fraction, except for PEB proteins. In PEB, only 234 out of the 1583 quantified proteins were classified as cytoskeletal proteins. PEB fraction contained cytoskeletal proteins, but also included insoluble high molecular weight proteins. 
Regarding global protein alterations in metastasis, we found more down- than upregulated proteins in the subcellular fractions of KM12SM highly metastatic cells. Using a 1.5-fold change, determined as significant by a permutation-based statistical test, we observed a total of 1318 altered proteins in the different subcellular compartments (Fig. 2D, Table S4). Collectively, western blot, MS and bioinformatics analyses results suggested an overall correct subcellular fractionation and the wide distribution of the proteins in multiple subcellular locations.

\subsection{Protein clusters, pathways and network analysis of differentially expressed proteins in CRC metastatic cells}

To characterize the metastatic behavior, we carried out a global in silico analysis of the differentially-expressed proteins in order to visualize protein-protein interactions and the most significantly altered protein clusters and macromolecular complexes using a circular layout algorithm with Cytoscape (Fig. 3, Table S5). Despite the high number of nodes and connections among the quantified proteins, the graphic representation highlighted significant networks and complexes. In the cytoplasmic compartment (CEB), GO annotation and network analysis showed a highly significant enrichment of "Translation initiation" and "Chaperonin-containing $T(C C T)$ complex" among the upregulated proteins (Fig. 3A), being EiF2 signaling one of the most significant canonical pathways affected ( $p$ : $\left.6.67 \times 10^{-8}\right)$. Regarding down-regulation, we observed significant alterations in "Vesicle-mediated transport" and "Small GTPase mediated signal transduction" (Fig. 3B).

In the membrane fraction (MEB), the SNARE complex, the "Ubiquinol cytochrome $c$ reductase complex core" and the "mitochondrial proton-transporting ATP synthase" complexes showed the highest enrichment among the up-regulated proteins (Fig. 3A). 
Among the down-regulated proteins, the "Proteasome complex" was highly enriched, as well as the "eukaryotic translation initiation factor", the "CCT complex" and “ribosomal proteins" (Fig. 3B).

In the nuclear compartment (NEB), whereas no significant networks or pathways were up-regulated (Fig. 3A), the "Arp2/3 complex", the "Proteasome complex" and the "MCM-complex" were down-regulated together with mitochondrial ribosomal proteins (Fig. 3B). In the chromatin-binding fraction (NEB-CBP), we noticed an unexpected enrichment of proteins implicated in the "Adherens Junction" and the "Tight Junction" pathway for the up-regulated proteins (Fig. 3A). For down-regulated proteins, the “replication fork" and "DNA replication” were highly enriched (Fig. 3B).

Finally, in the cytoskeletal compartment (PEB), the "COPI vesicle coat" and the "AP-type membrane coat adaptor complex" were highly prominent (Fig. 3A). In contrast, no significant networks and pathways were enriched within the 121 downregulated proteins (Fig. 3B). Interestingly, some protein complexes exhibited different alterations in abundance depending on the cellular localization.

\subsection{Mapping multidimensional protein-complex alterations in CRC metastatic cells}

Although $91 \%$ of the 1318 unique deregulated proteins showed similar alterations in the same or different subcellular compartments, 117 proteins (8.9\%) showed an opposite quantification in different subcellular localizations (Fig. 4, Table S6). Among protein complexes showing similar alterations in different subcellular compartments, the Arp2/3 complex, the replication fork, the MCM complex and the proteasome complex were the most down-regulated complexes. On the other hand, the most up-regulated complexes were those related to vesicle membrane trafficking, including the SNARE complex and 
proteins of the AP-type membrane coat adaptor complex and COPI vesicle coat (Fig. 4A, B).

Regarding the subset of 117 proteins showing opposite changes in expression (Fig. 4C), three main complexes were significantly altered in different subcellular compartments: the cytosolic chaperonin CCT/TRiC complex (CCT-complex), the mitochondrial proton-transporting ATP synthase, and the translation initiation -Eif2 and Eif3- complexes (Fig. 4C).

\subsection{Clustering and validation of spatially-deregulated proteins and their interactome}

To further investigate the distribution of the spatially deregulated proteins, the 117 proteins were subjected to unsupervised clustering according to their differential expression in the subcellular fractions (Fig. 5A) and protein-protein interaction analysis using STRING (Fig. 5B). STRING provided a vision of the "core" proteins of the complex and other interacting proteins. In general, the core proteins of the complex had similar expression patterns in different locations, with some exceptions like CCT2 or EIF4A1, which showed additional high expression associated to the cytoskeleton fraction (PEB). While CCT2 has been associated to tubulin, the cytoskeletal location would be novel for EIF4A1. Other interacting non-core proteins of the complex may present different trends in expression in different localizations (Fig. 5B). The 11 proteins related to cell adhesion, including CTNNB1, CTNNA1, TJP2/ZO2, MARCKS and FERMT1, showed more variability in localization and significant differences among them. These changes in location during cancer progression were well known for

$\alpha$ - and $\beta$-catenin, but they were novel for the other proteins. A potential explanation is that the network core proteins travel collectively as a complex to different locations, 
whereas the "non-core" interacting proteins may vary according to the organelle or subcellular fraction.

To verify the MS dataset and the in silico cluster analysis, we investigated protein expression of some representative complexes by western blot and confocal microscopy. By western blot, TCP1 and CCT2 from the CCT-complex showed similar alterations to MS ratios as well as G3BP1, GNB2 and Rab35 of the guanine-nucleotide binding proteins. A similar situation was observed for the adhesion proteins TLN1 and ZO1 proteins, which showed opposite expression in different compartments by MS and western blot (Fig. 5C). Cytoplasmic protein RhoGDi was included as loading control. Visualization by confocal microscopy confirmed MS results at the subcellular level (Fig. 6). An increased cytoplasmic expression of CCT2 and G3BP1 was observed in the metastatic KM12SM cells, with increased association to the cytoskeleton for CCT2 and some nuclear location for G3BP1. For RAB35, we noticed a predominant membrane location in metastatic cells, with reduced cytoplasmic staining. GTPase RAB35 plays an important role in endocytic recycling. In agreement with MS data, RAB35 was upregulated in MEB and down-regulated in other compartments in KM12SM cells, suggesting an implication of RAB35 in the formation of vesicles in highly metastatic cells. In summary, metastatic cells present changes in the spatio-temporal distribution of altered protein abundances, which would be impossible to detect without subcellular fractionation. These proteins might possess different functions in different localizations which might be relevant for CRC metastasis. 


\section{Discussion}

Here, we have performed a draft map of the spatial proteome in metastatic colorectal cancer cells. Subcellular fractionation enabled the characterization and quantification of 4863 proteins in different subcellular compartments. The deregulated proteome and their spatial distribution between the cytoplasm, membrane, nucleus, chromatin and cytoskeletal fractions were used to study global alterations in cancer metastatic cells. In total, 1318 proteins were deregulated in metastatic cells and $8.9 \%$ of deregulated proteins showed a multidimensional alteration. Spatial alterations were confirmed by western blot and confocal microscopy. To our knowledge, this study provides one of the widest quantitative analysis of multidimensional proteome alterations in CRC metastasis.

Most of the proteins and protein complexes showed similar expression trends in the different cellular compartments. The most up-regulated protein alterations in metastatic cells involved the SNARE complex, the AP-type membrane coat adaptor complex and the COPI vesicle coat related with the transport of vesicles. SNARE and other components of the fusion machinery seem to be involved in exosome secretion [36]. A higher exosome release has been associated with tumor progression and metastasis [37]. Previously, we reported that 88 out of 155 deregulated proteins found in the secretome of KM12 cells were exosome-related [20]. Therefore, alterations in the membrane fusion machinery of the highly metastatic cells would explain the increased vesicular trafficking and exosome release. In neuroinflammation and neurodegeneration processes [38, 39], it has been suggested an alternative use of exosomes as a compensatory mechanism to get rid of unwanted or unfolded proteins. Intracellular traffic of membrane vesicles is also responsible for the maintenance and regulation of the components of the plasma membrane [40]. Alterations in the accurate delivery of 
proteins to the cell surface can lead to the loss of cellular polarity, one of the earliest stages of carcinogenesis [41, 42], invasion and metastasis [43]. Thus, vesicle trafficking is central for understanding carcinogenesis and for developing novel therapeutic strategies. Proteins from the SNARE complex, like syntaxins, might be used as novel targets in cancer [44].

The most down-regulated processes in metastatic cells were those related to the proteasome complex, the ARP2/3 complex, the replication fork and the MCM complex. The ubiquitin-proteasome system is the major mechanism regulating protein turnover, and thus, cells are highly vulnerable to its malfunction [45]. We observed that 10 proteins of the 19S proteasome regulatory complex were down-regulated, including all six ATPase and four non-ATPase subunits. Interestingly, reduced expression of individual subunits of the proteasome's $19 \mathrm{~S}$ regulatory complex increases cell survival and drug-resistance of myeloma cells [46]. Our findings would support an increase in the survival and proteasome drug-resistance of highly metastatic CRC cells.

The ARP2/3 complex participates in the nucleation of actin and in the process of actin polymerization and depolymerization $[47,48]$. ARP2/3 complex dysfunction has been associated with the increased capability of metastatic cells to migrate away from primary tumors and invade healthy tissues [49]. Although usually present in the cytoskeleton and cytosol, some studies have demonstrated that the ARP2/3 complex is present in the nucleus, interacting with the NonO/PSF/N-WASP/RNA POL II complex to regulate transcription and chromatin organization $[50,51]$. The observed down regulation of the ARP2/3 complex in the nuclear compartment might be related with a lower presence of actin in the nucleus due to higher cytoplasmic requirements [51].

Interestingly, 117 proteins showed multidimensional dysregulation in expression and localization. Many of these proteins were forming large macromolecular complexes. 
For example, we found spatially-dependent alterations in the MS ratios of 7 out of 8 proteins of the chaperonin CCT-complex, changing the MS ratio from 2 in CEB to 0.5 in MEB. The CCT/TRiC complex seems to play a key role in cell cycle progression and tumor development [52]. An analysis of the interaction network of the CCT chaperonins revealed a high relevance in the folding of many proteins, including actin and tubulin [53]. Indeed, CCT targets can be divided in ATP-dependent or independent. ARP $2 / 3$ proteins were ATP independent, whereas mitochondrial and other membrane proteins required ATP for the interaction with CCT [53]. This ATP-dependence might explain the differential expression of the CCT complex between the CEB fraction and the MEB fraction, enriched in cytosolic and mitochondrial and ribosomal proteins, respectively.

Sixteen proteins of the mitochondrial proton-transporting ATP synthase complexes, ABCB7, ATP5B, ATP5C1, ATP5D, ATP5F1, ATP5J2, ATP5O, COX6C, COX7A2, and DLST, associated to the oxidative phosphorylation (OxPhos) were mostly upregulated in MEB compartment (mitochondrial localization). However, ATP5A1, ATP5H, UQCRC1, UQCRC2, and UQCRFS1, including three proteins of the Cytochrome b-c1 mitochondrial complex, were reduced in the cytoskeleton compartment (PEB). These data -apart from indicating a mitochondrial dysfunction (as also suggested by pathway analysis)- suggest that proteins belonging to the TCA and OxPhos processes might have dual cell localizations with different functions in these secondary localizations (nucleus and cytoskeleton), as reported in MCF7 breast cancer cells [54].

Proteins from the heterotrimeric-G protein complex (GNA11, GNB1, GNB2, and GNAI3), GTPases (RAB35, SRP54, and RHOG) or GTPase-activating proteins (ARFGAP2 and ARHGAP18) were up-regulated in the MEB fraction (plasma, mitochondria and ER/golgi membranes). However, they were down-regulated in the 
CEB (cytoplasm) compartment in metastatic cells. Proteins of this complex are localized to different organelles and they cooperate with the SNARE proteins for vesicle docking and the fusion machinery. RAB GTPases are probably sequestered from the cytosol and moved to the membrane compartment, where they control the SNARE complex formation and are involved in exosome secretion [36]. Thus, it is consequent that RAB GTPases (i.e. RAB35) are upregulated in the MEB compartment, as well as the SNARE proteins, and down-regulated in the CEB compartment. In particular, RAB35 mediates slow endocytic recycling through recycling endosome to the basolateral plasma membrane [55], and has been involved also in the late stages of cytokinesis [56]. RAB35 and RAB27A/B allowed the docking of vesicular bodies to the plasma membrane [57]. Therefore, RAB35 might constitute a new potential therapeutic target in colon cancer metastasis, as has been suggested for other GTPases and GTPaseactivating proteins in melanoma or lung cancer [58].

Dysregulation of cell junction adhesion proteins has been heavily implicated in the epithelial-mesenchymal transition that leads to metastasis [59]. For instance, metastatic breast cancer cells express relatively little $\mathrm{ZO}-1$ protein [60]. In this group of proteins, there was not a homogeneous distribution pattern. Whereas our results confirm the translocation of $\beta$-catenin to the nucleus (NEB-CBP) associated to cancer progression, $\alpha$-catenin showed increased association to actin cytoskeleton (PEB). TJP2, aka ZO2, a tight junction protein was detected at the nucleus and the cytoskeleton. This interesting finding supports a multifunctional role for this protein in chromatin organization and transcriptional control in cancer metastatic cells [61]. We also confirm the recent nuclear assignment of MARCKS and its potential function in regulation of gene expression [62], indicating another dual localization for this adhesion protein. 
In summary, we quantified deregulated proteins and mapped its localization in metastatic colorectal cancer cells. Our data highlight the importance of protein localization to distinguish proteins and complexes that behave differently in different organelles and locations in order to identify underlying mechanisms of CRC metastasis. Subcellular alterations of proteins are a characteristic of metastatic cells that may be vital for cancer progression and metastasis, including exosome secretion. In addition, this multidimensional information has provided a new panel of potential subcellular biomarkers and targets for intervention in cancer metastasis.

\section{Conflict of interest}

The authors declare no conflict of interest

\section{Acknowledgements}

This research was supported by grants BIO2012-31023 and BIO2015-66489-R from the MINECO, grant PRB2 (IPT13/0001-ISCIII-SGEFI/FEDER) and Foundation Ramón Areces to JIC. Work at RB's lab was supported by a grant from the MINECO (SAF2014-53209-R). 


\section{References}

[1] Larance, M., Lamond, A. I., Multidimensional proteomics for cell biology. Nat Rev Mol Cell Biol 2015, 16, 269-280.

[2] Henke, R. M., Dastidar, R. G., Shah, A., Cadinu, D., et al., Hypoxia elicits broad and systematic changes in protein subcellular localization. Am J Physiol Cell Physiol 2011, 301, C913-928.

[3] Chu, H. Y., Hopper, A. K., Genome-wide investigation of the role of the tRNA nuclear-cytoplasmic trafficking pathway in regulation of the yeast Saccharomyces cerevisiae transcriptome and proteome. Mol Cell Biol 2013, 33, 4241-4254.

[4] Fabre, B., Lambour, T., Delobel, J., Amalric, F., et al., Subcellular distribution and dynamics of active proteasome complexes unraveled by a workflow combining in vivo complex cross-linking and quantitative proteomics. Mol Cell Proteomics 2013, 12, 687699.

[5] Lamond, A. I., Uhlen, M., Horning, S., Makarov, A., et al., Advancing cell biology through proteomics in space and time (PROSPECTS). Mol Cell Proteomics 2012, 11, O112017731.

[6] Kimura, M., Kose, S., Okumura, N., Imai, K., et al., Identification of cargo proteins specific for the nucleocytoplasmic transport carrier transportin by combination of an in vitro transport system and stable isotope labeling by amino acids in cell culture (SILAC)-based quantitative proteomics. Mol Cell Proteomics 2013, 12, 145-157. [7] Sorokin, A. V., Kim, E. R., Ovchinnikov, L. P., Nucleocytoplasmic transport of proteins. Biochemistry (Mosc) 2007, 72, 1439-1457.

[8] Baqader, N. O., Radulovic, M., Crawford, M., Stoeber, K., Godovac-Zimmermann, J., Nuclear cytoplasmic trafficking of proteins is a major response of human fibroblasts to oxidative stress. J Proteome Res 2014, 13, 4398-4423.

[9] Boisvert, F. M., Lam, Y. W., Lamont, D., Lamond, A. I., A quantitative proteomics analysis of subcellular proteome localization and changes induced by DNA damage. Mol Cell Proteomics 2010, 9, 457-470.

[10] Park, S., Yang, J. S., Shin, Y. E., Park, J., et al., Protein localization as a principal feature of the etiology and comorbidity of genetic diseases. Mol Syst Biol 2011, 7, 494. [11] Sirover, M. A., Subcellular dynamics of multifunctional protein regulation: mechanisms of GAPDH intracellular translocation. J Cell Biochem 2012, 113, 21932200. 
[12] Tristan, C., Shahani, N., Sedlak, T. W., Sawa, A., The diverse functions of GAPDH: views from different subcellular compartments. Cell Signal 2011, 23, 317323.

[13] Laurila, K., Vihinen, M., Prediction of disease-related mutations affecting protein localization. BMC Genomics 2009, 10, 122.

[14] Kau, T. R., Way, J. C., Silver, P. A., Nuclear transport and cancer: From mechanism to intervention. Nature Reviews Cancer 2004, 4, 106-117.

[15] Siegel, R., Desantis, C., Jemal, A., Colorectal cancer statistics, 2014. CA Cancer J Clin 2014, 64, 104-117.

[16] Valastyan, S., Weinberg, R. A., Tumor metastasis: molecular insights and evolving paradigms. Cell 2011, 147, 275-292.

[17] Fidler, I. J., The pathogenesis of cancer metastasis: the 'seed and soil' hypothesis revisited. Nat Rev Cancer 2003, 3, 453-458.

[18] Morikawa, K., Walker, S. M., Jessup, J. M., Fidler, I. J., In vivo selection of highly metastatic cells from surgical specimens of different primary human colon carcinomas implanted into nude mice. Cancer Res 1988, 48, 1943-1948.

[19] Barderas, R., Bartolome, R. A., Fernandez-Acenero, M. J., Torres, S., Casal, J. I., High expression of IL-13 receptor alpha2 in colorectal cancer is associated with invasion, liver metastasis, and poor prognosis. Cancer Res 2012, 72, 2780-2790. [20] Barderas, R., Mendes, M., Torres, S., Bartolome, R. A., et al., In-depth characterization of the secretome of colorectal cancer metastatic cells identifies key proteins in cell adhesion, migration, and invasion. Mol Cell Proteomics 2013, 12, $1602-$ 1620.

[21] Luque-Garcia, J. L., Martinez-Torrecuadrada, J. L., Epifano, C., Canamero, M., et $a l .$, Differential protein expression on the cell surface of colorectal cancer cells associated to tumor metastasis. Proteomics 2010, 10, 940-952.

[22] Boisvert, F. M., Ahmad, Y., Gierlinski, M., Charriere, F., et al., A quantitative spatial proteomics analysis of proteome turnover in human cells. Mol Cell Proteomics 2012, 11, M111 011429.

[23] Christoforou, A., Mulvey, C. M., Breckels, L. M., Geladaki, A., et al., A draft map of the mouse pluripotent stem cell spatial proteome. Nat Commun 2016, 7, 8992. [24] Morikawa, K., Walker, S. M., Nakajima, M., Pathak, S., et al., Influence of organ environment on the growth, selection, and metastasis of human colon carcinoma cells in nude mice. Cancer Res 1988, 48, 6863-6871. 
[25] Pelaez-Garcia, A., Barderas, R., Batlle, R., Vinas-Castells, R., et al., A proteomic analysis reveals that Snail regulates the expression of the nuclear orphan receptor Nuclear Receptor Subfamily 2 Group F Member 6 (Nr2f6) and interleukin 17 (IL-17) to inhibit adipocyte differentiation. Mol Cell Proteomics 2015, 14, 303-315.

[26] Chen, D., Shah, A., Nguyen, H., Loo, D., et al., Online quantitative proteomics pvalue calculator for permutation-based statistical testing of peptide ratios. J Proteome Res 2014, 13, 4184-4191.

[27] Mi, H., Muruganujan, A., Casagrande, J. T., Thomas, P. D., Large-scale gene function analysis with the PANTHER classification system. Nat Protoc 2013, 8, 15511566.

[28] Medina, I., Carbonell, J., Pulido, L., Madeira, S. C., et al., Babelomics: an integrative platform for the analysis of transcriptomics, proteomics and genomic data with advanced functional profiling. Nucleic Acids Res 2010, 38, W210-213. [29] Huang da, W., Sherman, B. T., Lempicki, R. A., Systematic and integrative analysis of large gene lists using DAVID bioinformatics resources. Nat Protoc 2009, 4, 44-57.

[30] Franceschini, A., Szklarczyk, D., Frankild, S., Kuhn, M., et al., STRING v9.1: protein-protein interaction networks, with increased coverage and integration. Nucleic Acids Res 2013, 41, D808-815.

[31] Kanehisa, M., Goto, S., Sato, Y., Kawashima, M., et al., Data, information, knowledge and principle: back to metabolism in KEGG. Nucleic Acids Res 2014, 42, D199-205.

[32] Warde-Farley, D., Donaldson, S. L., Comes, O., Zuberi, K., et al., The GeneMANIA prediction server: biological network integration for gene prioritization and predicting gene function. Nucleic Acids Res 2010, 38, W214-220.

[33] Agapito, G., Guzzi, P. H., Cannataro, M., Visualization of protein interaction networks: problems and solutions. BMC Bioinformatics 2013, 14 Suppl 1, S1.

[34] Cline, M. S., Smoot, M., Cerami, E., Kuchinsky, A., et al., Integration of biological networks and gene expression data using Cytoscape. Nat Protoc 2007, 2, 2366-2382. [35] Babel, I., Barderas, R., Diaz-Uriarte, R., Martinez-Torrecuadrada, J. L., et al., Identification of tumor-associated autoantigens for the diagnosis of colorectal cancer in serum using high density protein microarrays. Mol Cell Proteomics 2009, 8, 2382-2395. 
[36] Colombo, M., Raposo, G., Thery, C., Biogenesis, secretion, and intercellular interactions of exosomes and other extracellular vesicles. Annu Rev Cell Dev Biol 2014, 30, 255-289.

[37] Ginestra, A., Miceli, D., Dolo, V., Romano, F. M., Vittorelli, M. L., Membrane vesicles in ovarian cancer fluids: A new potential marker. Anticancer Research 1999, $19,3439-3445$.

[38] Brites, D., Fernandes, A., Neuroinflammation and Depression: Microglia Activation, Extracellular Microvesicles and microRNA Dysregulation. Front Cell Neurosci 2015, 9, 476.

[39] Joshi, P., Benussi, L., Furlan, R., Ghidoni, R., Verderio, C., Extracellular vesicles in Alzheimer's disease: friends or foes? Focus on abeta-vesicle interaction. Int J Mol Sci 2015, 16, 4800-4813.

[40] Mellman, I., Nelson, W. J., Coordinated protein sorting, targeting and distribution in polarized cells. Nat Rev Mol Cell Biol 2008, 9, 833-845.

[41] Royer, C., Lu, X., Epithelial cell polarity: a major gatekeeper against cancer? Cell Death Differ 2011, 18, 1470-1477.

[42] McCaffrey, L. M., Macara, I. G., Epithelial organization, cell polarity and tumorigenesis. Trends Cell Biol 2011, 21, 727-735.

[43] Goldenring, J. R., A central role for vesicle trafficking in epithelial neoplasia: intracellular highways to carcinogenesis. Nat Rev Cancer 2013, 13, 813-820.

[44] Riggs, K. A., Hasan, N., Humphrey, D., Raleigh, C., et al., Regulation of integrin endocytic recycling and chemotactic cell migration by syntaxin 6 and VAMP3 interaction. J Cell Sci 2012, 125, 3827-3839.

[45] Petrocca, F., Altschuler, G., Tan, S. M., Mendillo, M. L., et al., A Genome-wide siRNA Screen Identifies Proteasome Addiction as a Vulnerability of Basal-like TripleNegative Breast Cancer Cells. Cancer Cell 2013, 24, 182-196.

[46] Acosta-Alvear, D., Cho, M. Y., Wild, T., Buchholz, T. J., et al., Paradoxical resistance of multiple myeloma to proteasome inhibitors by decreased levels of $19 \mathrm{~S}$ proteasomal subunits. Elife 2015, 4, e08153.

[47] Goley, E. D., Welch, M. D., The ARP2/3 complex: an actin nucleator comes of age. Nat Rev Mol Cell Biol 2006, 7, 713-726.

[48] Korobova, F., Svitkina, T., Arp2/3 complex is important for filopodia formation, growth cone motility, and neuritogenesis in neuronal cells. Molecular Biology of the Cell 2008, 19, 1561-1574. 
[49] Wang, W., Goswami, S., Sahai, E., Wyckoff, J. B., et al., Tumor cells caught in the act of invading: their strategy for enhanced cell motility. Trends Cell Biol 2005, 15, 138-145.

[50] Yoo, Y., Wu, X., Guan, J. L., A novel role of the actin-nucleating Arp2/3 complex in the regulation of RNA polymerase II-dependent transcription. J Biol Chem 2007, 282, 7616-7623.

[51] Gieni, R. S., Hendzel, M. J., Actin dynamics and functions in the interphase nucleus: moving toward an understanding of nuclear polymeric actin. Biochem Cell Biol 2009, 87, 283-306.

[52] Boudiaf-Benmammar, C., Cresteil, T., Melki, R., The cytosolic chaperonin CCT/TRiC and cancer cell proliferation. PLoS One 2013, 8, e60895.

[53] Dekker, C., Stirling, P. C., McCormack, E. A., Filmore, H., et al., The interaction network of the chaperonin CCT. EMBO J 2008, 27, 1827-1839.

[54] Qattan, A. T., Radulovic, M., Crawford, M., Godovac-Zimmermann, J., Spatial distribution of cellular function: the partitioning of proteins between mitochondria and the nucleus in MCF7 breast cancer cells. J Proteome Res 2012, 11, 6080-6101. [55] Stenmark, H., Rab GTPases as coordinators of vesicle traffic. Nat Rev Mol Cell Biol 2009, 10, 513-525.

[56] Kouranti, I., Sachse, M., Arouche, N., Goud, B., Echard, A., Rab35 regulates an endocytic recycling pathway essential for the terminal steps of cytokinesis. Curr Biol 2006, 16, 1719-1725.

[57] Hsu, C., Morohashi, Y., Yoshimura, S., Manrique-Hoyos, N., et al., Regulation of exosome secretion by Rab35 and its GTPase-activating proteins TBC1D10A-C. J Cell Biol 2010, 189, 223-232.

[58] Gysin, S., Salt, M., Young, A., McCormick, F., Therapeutic strategies for targeting ras proteins. Genes Cancer 2011, 2, 359-372.

[59] Martin, T. A., Jiang, W. G., Loss of tight junction barrier function and its role in cancer metastasis. Biochim Biophys Acta 2009, 1788, 872-891.

[60] Hoevel, T., Macek, R., Mundigl, O., Swisshelm, K., Kubbies, M., Expression and targeting of the tight junction protein CLDN1 in CLDN1-negative human breast tumor cells. J Cell Physiol 2002, 191, 60-68.

[61] Traweger, A., Lehner, C., Farkas, A., Krizbai, I. A., et al., Nuclear Zonula occludens-2 alters gene expression and junctional stability in epithelial and endothelial cells. Differentiation 2008, 76, 99-106. 
[62] Rohrbach, T. D., Shah, N., Jackson, W. P., Feeney, E. V., et al., The Effector Domain of MARCKS Is a Nuclear Localization Signal that Regulates Cellular PIP2 Levels and Nuclear PIP2 Localization. PLoS One 2015, 10, e0140870. 


\section{Legend to Figures}

Figure 1. Subcellular fractionation and differential protein expression in KM12 cells. (A) For metabolic labeling, KM12C or KM12SM were grown and maintained in light and heavy-labeled SILAC DMEM medium supplemented with 10\% dialyzed FBS. Label swapping (forward and reverse) SILAC experiments were biochemically fractionated into five subcellular fractions corresponding to: cytoplasm (CEB), plasma, mitochondria and ER/golgi membranes (MEB), nuclear soluble (NEB), nuclear chromatin-bound (NEB-CBP) and cytoskeletal (PEB) proteins. Protein extracts were separated by SDS-PAGE. Mass spectra were acquired on an LTQ-Orbitrap Velos mass spectrometer. (B) Verification of the correct subcellular fractionation by western blot using antibodies against proteins of known subcellular localization. (C) The abundance of the proteins determined by MS was compared with western blot results. The relative average quantification of the forward and reverse peak areas obtained with Proteome Discoverer is depicted for each protein in each subcellular fraction. Values were normalized using the top area value in one of the 5 subcellular fractions as 1 . The rest of the values were calculated accordingly.

Figure 2. Analysis of the identified, quantified and deregulated proteins in KM12 cells. (A) Summary of the identified and quantified peptides and proteins in both SILAC experiments for the five subcellular fractions. (B) Venn diagram of the proteins quantified in CEB, MEB, NEB, NEB-CBP and PEB. (C) GO analysis for the subcellular classification of the proteins in each compartment was made with PANTHER. The top 5 subcellular GO classifications in each fraction are shown with the calculated correlation value. (D) Venn diagram of deregulated proteins showing a fold-change $\geq 1.5$. 
Figure 3. Deregulated protein networks, macromolecular complexes, and pathways in CRC metastatic cells. STRING, KEGG and IPA bioinformatics suites were used for in silico analysis. (A) up-regulated and (B) down-regulated proteinprotein interactions and networks of interest in KM12 cells with different metastatic properties. STRING was used to determine protein-protein interactions of the deregulated proteins. Then, data were exported to Cytoscape for the representation of deregulated alterations in all five subcellular fractions. Dashed lines correspond to the protein-protein interactions of deregulated proteins identified by STRING. Red: upregulated proteins, green: down-regulated proteins in KM12SM cells. Light to dark (green and red) colors and spot size are proportional to the fold-change variation observed for each protein in each subcellular fraction. Cytoscape was used for representation of deregulated alterations in all five subcellular fractions according to a circular layout algorithm.

Figure 4. Summary of subcellular alterations in protein networks and macromolecular complexes in CRC metastasis. (A) In total, we observed 1318 deregulated proteins, with $\sim 60 \%$ of the proteins showing downregulation, and 117 proteins an opposite regulation in different compartments. (B) Among the downregulated proteins, we mainly observed downregulation in the replication fork and in the proteasome, ARP2/3, and MCM complexes. Among the up-regulated proteins, we mainly observed upregulation in the SNARE complex and the COPI vesicle coat and AP-type membrane coat adaptor complex. (C) Proteins with opposite changes in expression in different subcellular compartments. Methods for data visualization were similar to the figure 3. 


\section{Figure 5. Hierarchical clustering and validation of proteins showing}

multidimensional alterations. (A) Unsupervised cluster analysis of the complete set of 117 proteins altered in expression. Green, down-regulation, and red, over-expression in KM12SM highly metastatic cells. Color scale is related to the fold-change observed for each protein in each subcellular fraction. (B) The 117 proteins were analyzed by STRING and IPA to find protein-protein interacting partners, and protein complexes. Then, they were separately analyzed by hierarchical clustering, and visualized through protein clusters by using STRING. In the cluster, green showed down-regulation, and red, over-expression in KM12SM cells for the indicated proteins. For a better visualization, fold-change values (rows) were normalized and the color scale set up between 0.16 (green) and 1.3 (red). Complex core proteins are encircled by a red line. (C) $10 \mu \mathrm{g}$ of the indicated total extracts or subcellular fractions of KM12C and KM12SM were subjected to western blot analysis and quantified by densitometry. Rho GDi was used as a control.

Figure 6. Confocal microscopy analysis of differential protein localization between KM12C and KM12SM cells. KM12C and KM12SM cell were cultured on glass coverslips pretreated with Matrigel for $24 \mathrm{~h}$ and subjected to confocal microscopy analysis using antibodies for CCT2, G3BP1 and RAB35 (green). Cells were counterstained with the nuclear probe DAPI (blue) and the actin cytoskeleton probe Phalloidin (red). Representative images show a differential staining distribution in the different cellular compartments for the three proteins between the metastatic and nonmetastatic cells. 


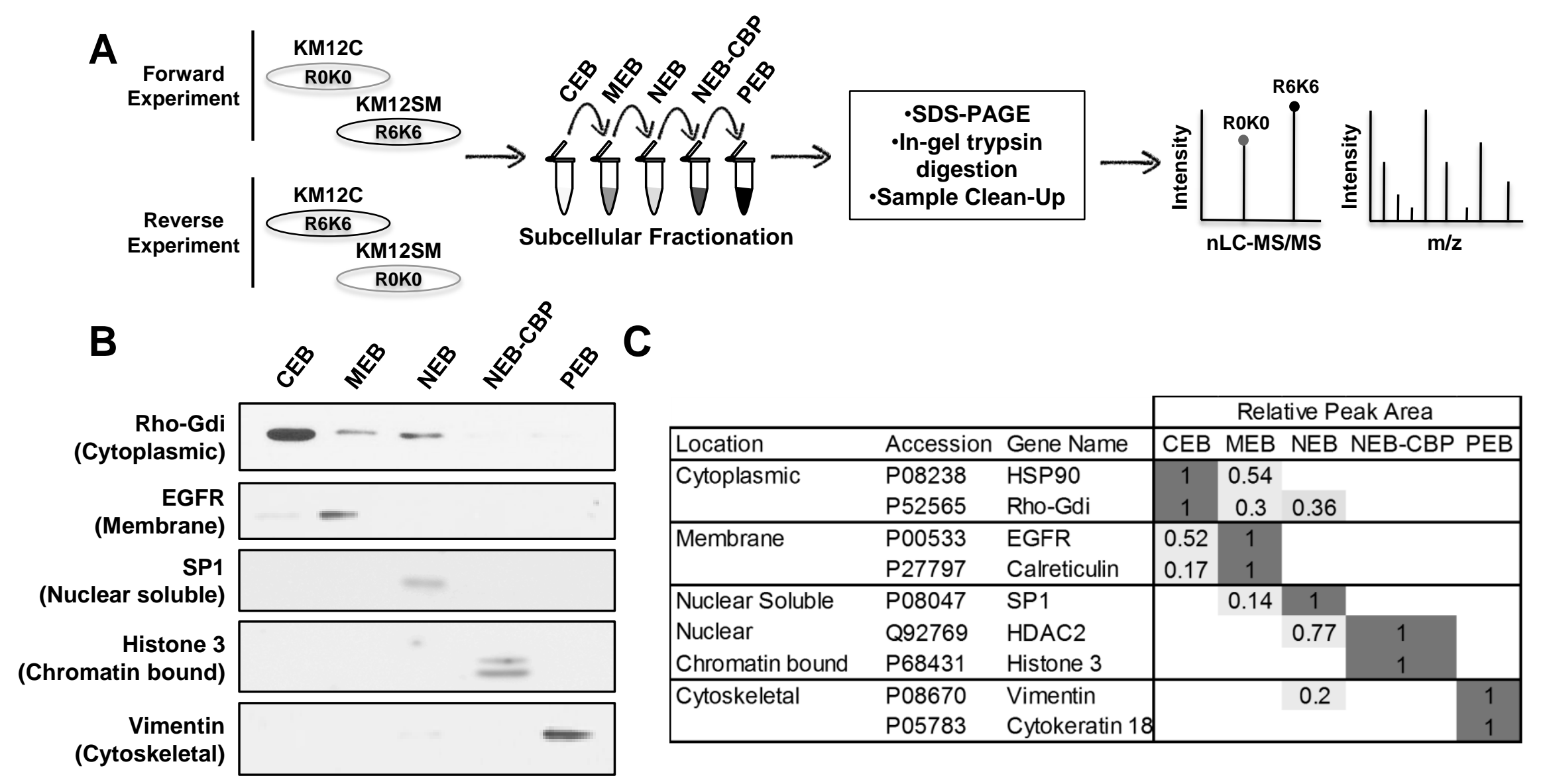

Figure 1 


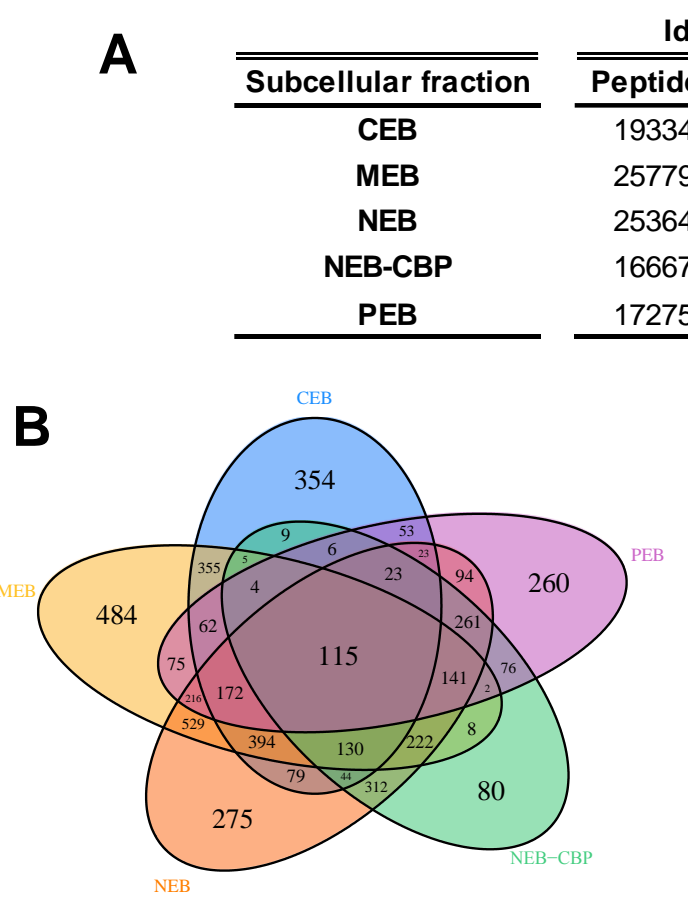

Quantified proteins
Identified

\begin{tabular}{|cc}
\hline tides & Proteins \\
\hline
\end{tabular}

$25779 \quad 4086$

3836

$6667 \quad 1853$

2117

\begin{tabular}{cc}
\multicolumn{2}{c}{ Quantified } \\
\hline \hline Peptides & Proteins \\
\hline 16519 & 1828 \\
24178 & 2914 \\
23435 & 3030 \\
14689 & 1483 \\
15306 & 1583 \\
\hline
\end{tabular}

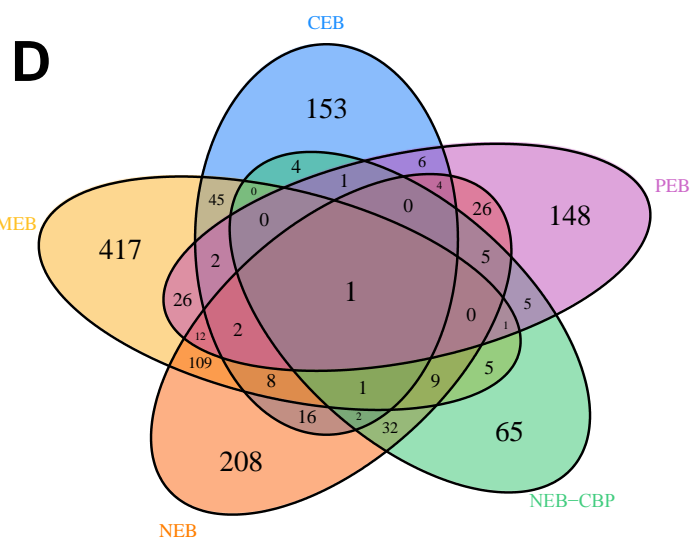

1.5-fold deregulated proteins

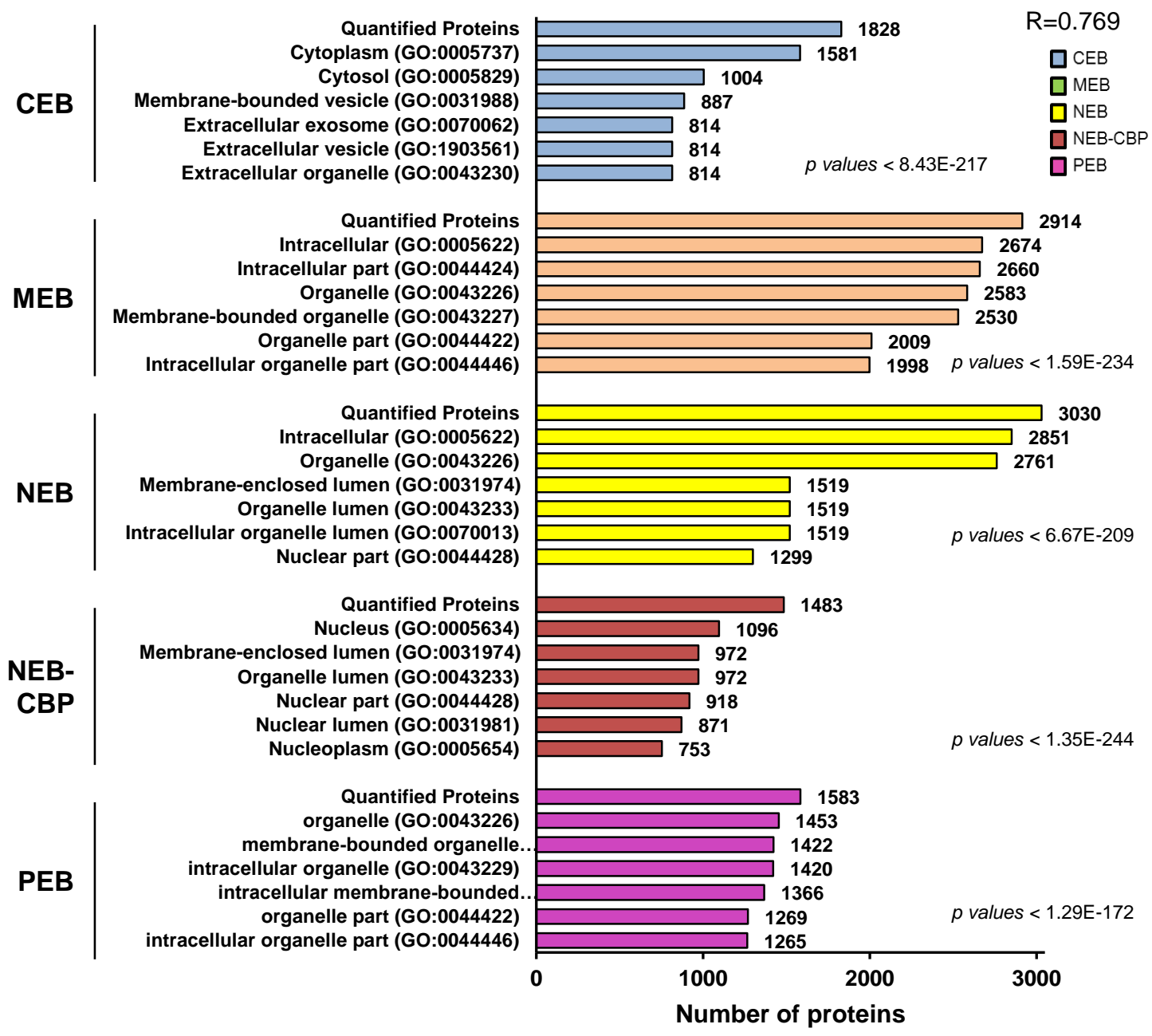


A Up-regulated pathways and protein complexes in CRC metastasis
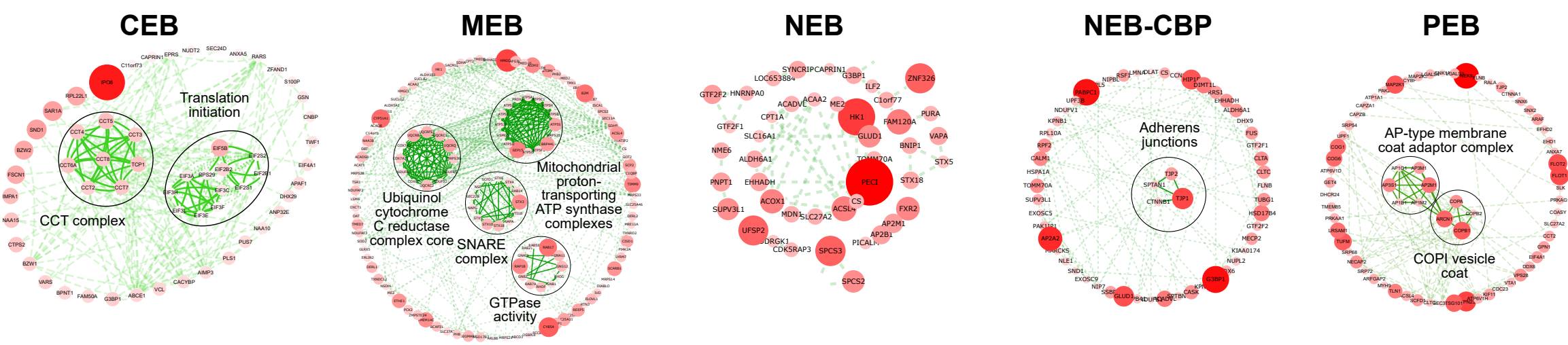

B Down-regulated pathways and protein complexes in CRC metastasis
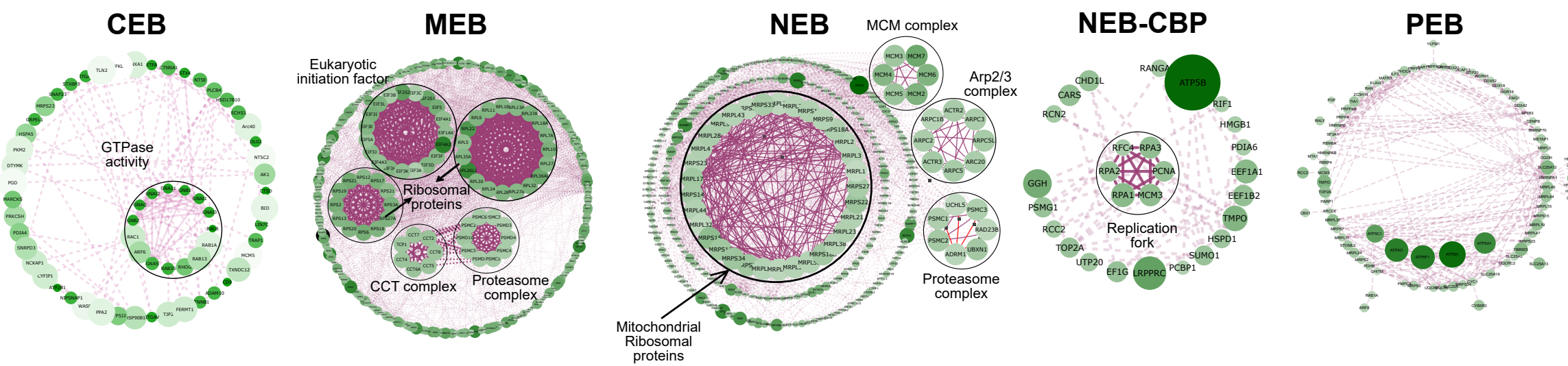
A

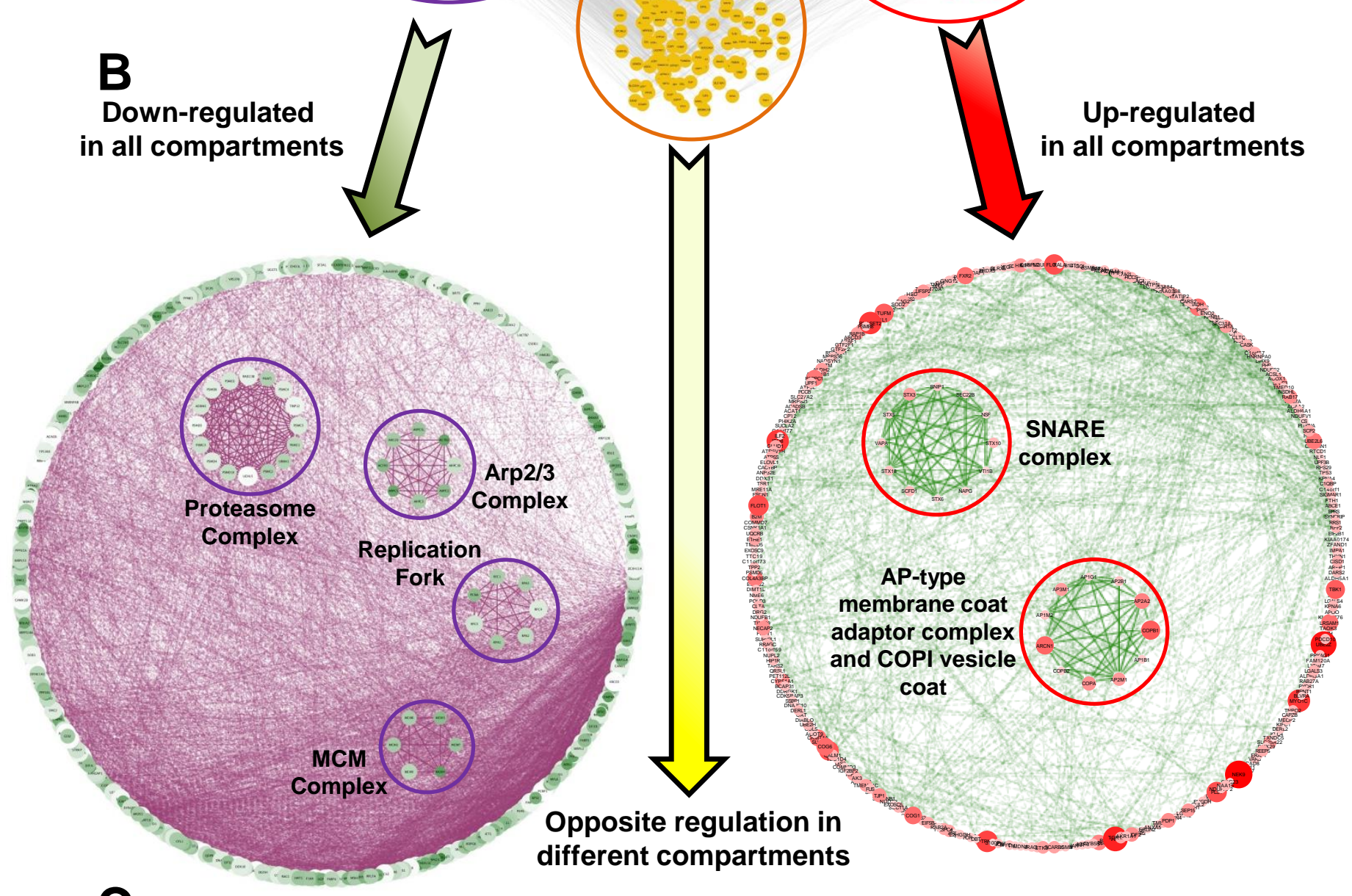

C
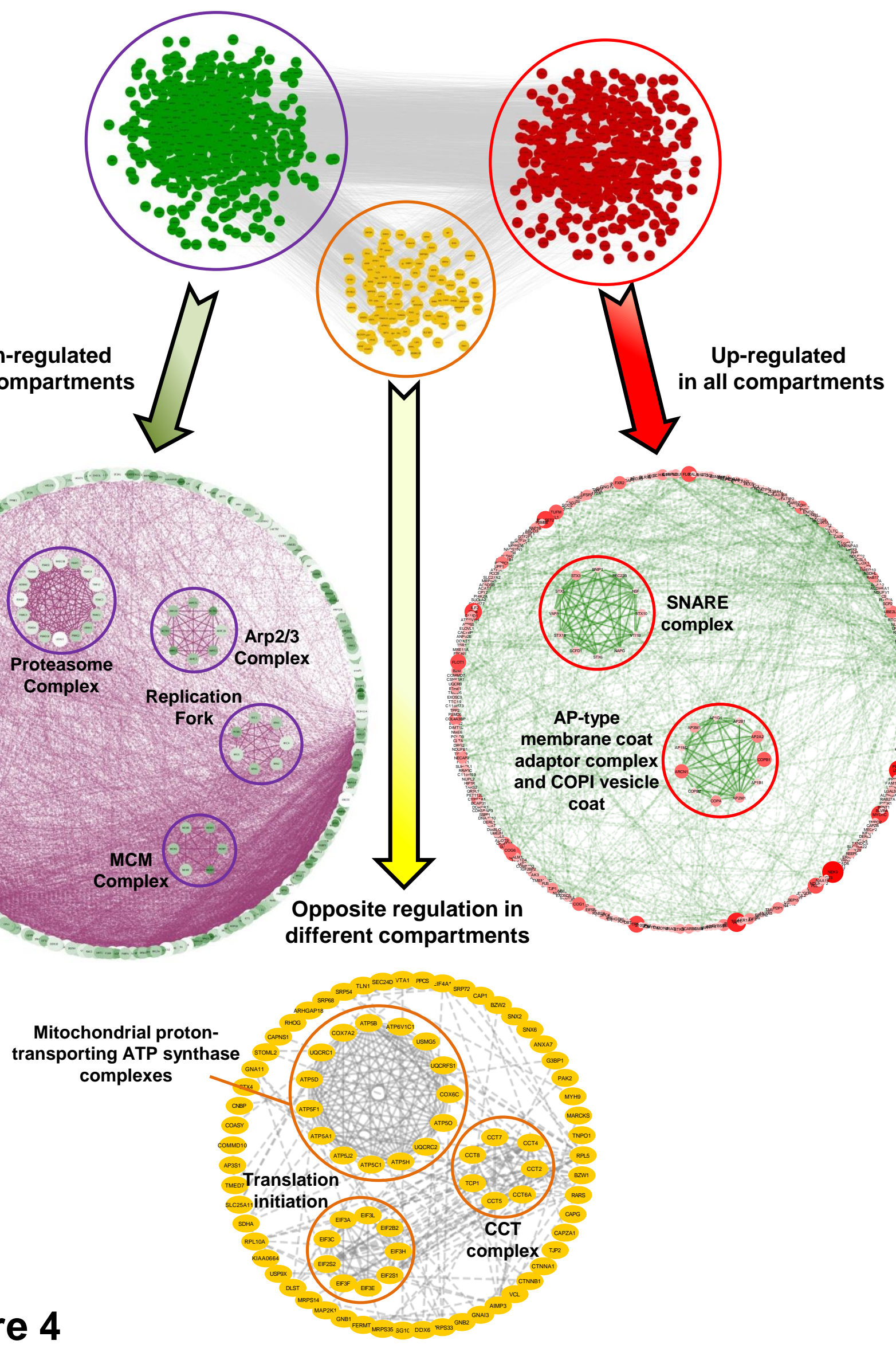

Figure 4 
A $\quad 0.160 .05347$

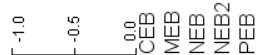

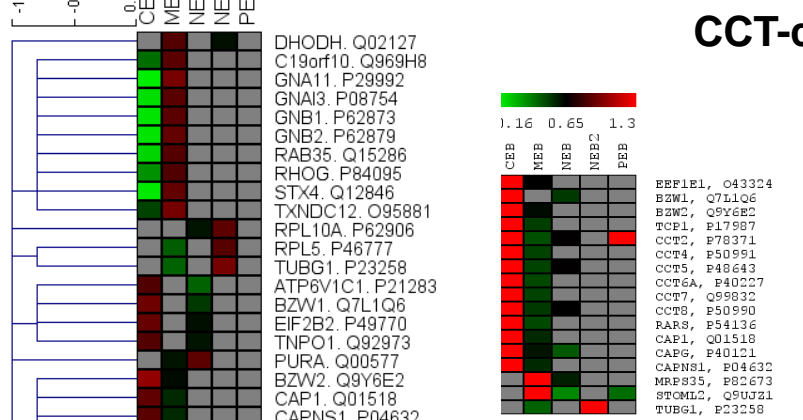

CCT-complex, stress fiber proteins and tubulin

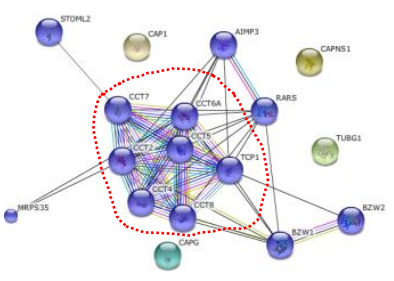

Eif2 and Eif3 complex
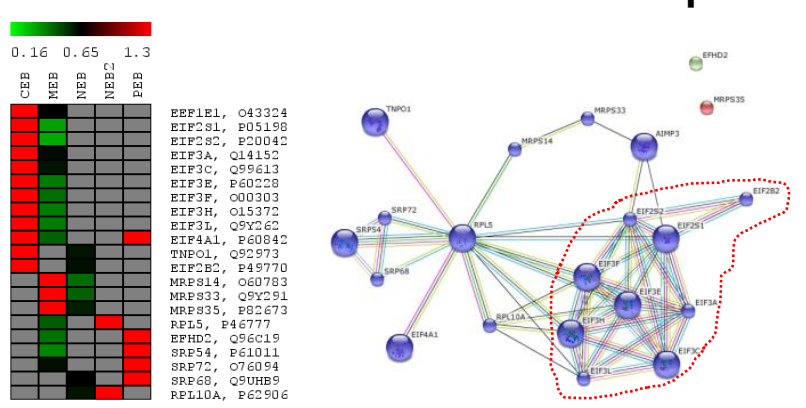

Oxidative Phosphorylation
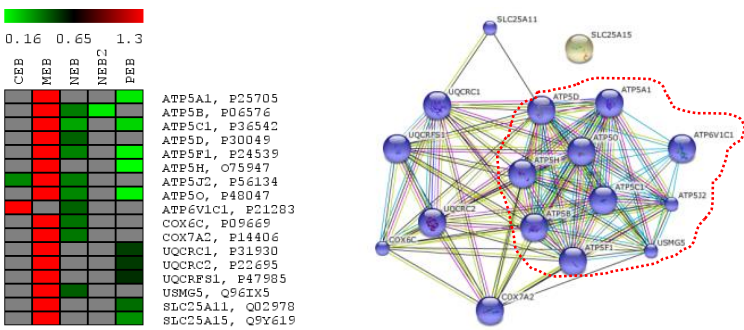

Guanine-nucleotide binding proteins
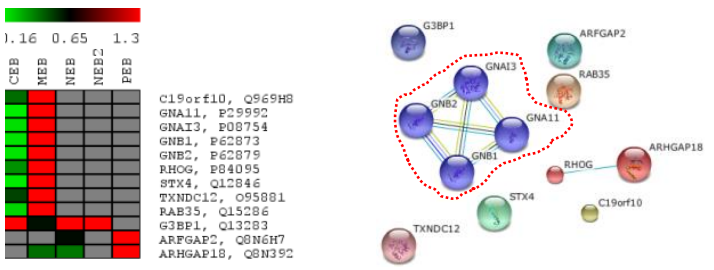

Adherens junctions

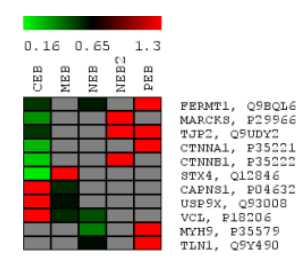

C

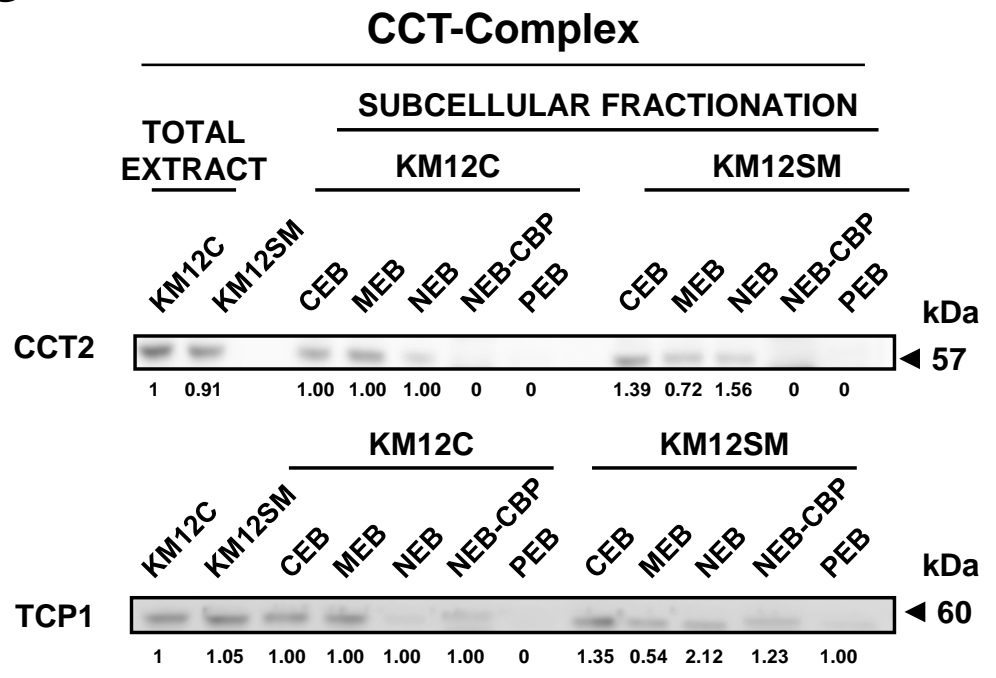

Adherens junctions

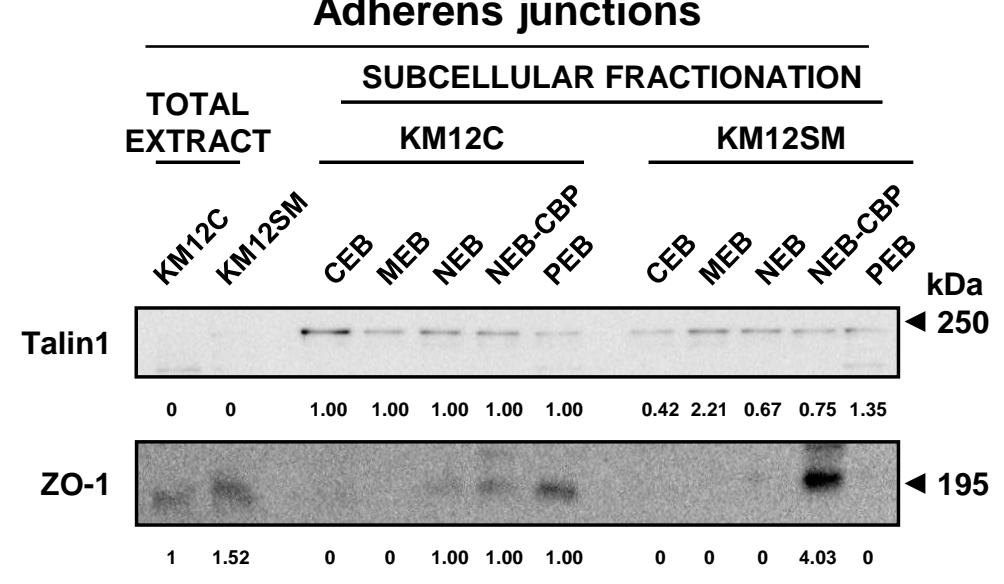

Guanine-nucleotide binding proteins
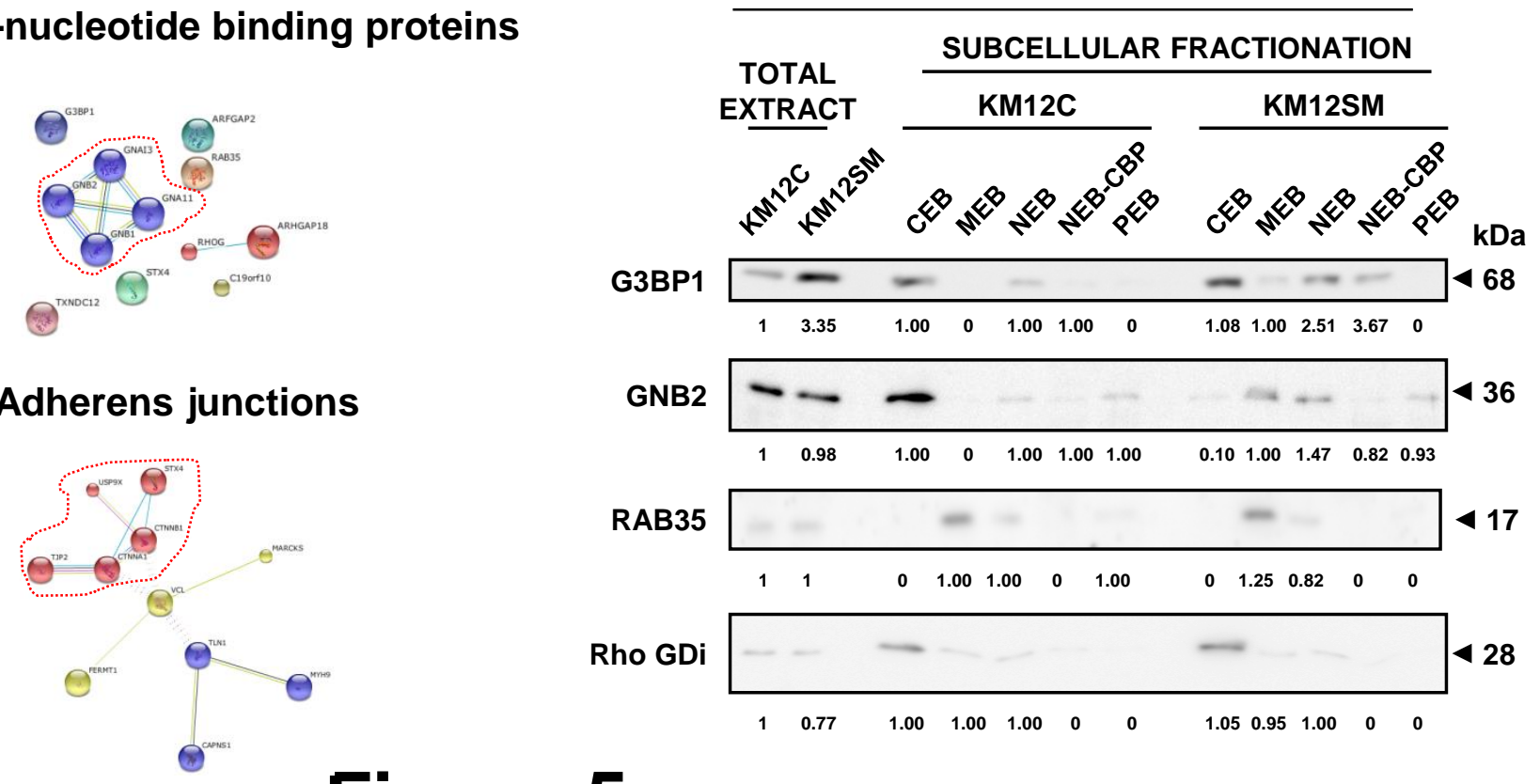
CCT2


Figure 6 